\title{
Interactive comment on "Assessment of cloud properties from the reanalysis with satellite observations over East Asia” by B. Yao et al.
}

\section{Anonymous Referee \#3}

Received and published: 22 December 2019

General Comments: This paper is about assessment of cloud properties from the reanalysis with satellite data over East Asia. Three sets of reanalysis data are used, including the newly developed China Meteorological Administration Reanalysis data (CRA), the ECMWF's Fifth-generation Reanalysis (ERA5), and the Modern-Era Retrospective Analysis for Applications, Version 2 (MERRA-2). And, to avoid the unrealistic assumptions and uncertainties on satellite retrieval algorithms and products, a radiative transfer model (CRTM) is used to transform reanalysis data into radiance/brightness temperature that can be directly compared with the Himawari-8 satellite data. Although cloud properties from CRA, ERA5, and MERRA-2 have their own advantages, the results show that ERA5 reanalysis data is best representative of cloudy atmosphere over East Asia, while the results in CRA are close to those in ERA5. This study may con- 
tribute to the improvement of cloudy property representation in models and satellite observations. This paper is within the scope of Atmospheric Measurement Techniques but some improvement should be conducted before the paper could be accepted for publication.

Major concerns: 1. The authors claim that the radiance-based evaluation approach could avoid unrealistic assumptions and uncertainties on satellite retrieval algorithms and products, and thus it is a better way to carry out the assessment of cloud properties from various reanalysis. However, I would say I only partially agree with the authors on the perspective that the conventional way to compare cloud variables could be still indispensable. Without knowing the quantitative and qualitative differences in cloud properties, it is still hard to explain the radiance/brightness temperature differences resulting from the radiative transfer modeling. Thus, more discussion about the cloud optical properties should be added. 2. Previous studies (i.e., Yi et al., JGR, 2017a, b) indicate that a consistent cloud optical property parameterization scheme should be used in satellite retrievals and modeling studies to well simulate the radiance/flux at the top of the atmosphere under cloudy sky. Any mismatch in cloud optics parameterization could induce large bias in the retrieval and simulations. Taking that into account, it seems the study here using CRTM with a new set of cloud optical property look up tables (it is also not clear what kind of ice cloud particle model is used) that is inconsistent with the Himawari-8 cloud retrieval algorithm, could be potentially problematic in the satellite radiance/brightness temperature simulation. The authors may need to consider using the Voronoi ice scattering model by Letu et al. $(2016 ; 2018)$. 3. Apart from the potential problem in cloud optical property, another important issue is about the differences in the atmospheric profiles. The simulated radiance/brightness temperature is closely related with the atmospheric profiles. Whereas, differences in the atmospheric profiles among the reanalysis datasets are prevalent. And these differences may contribute to the simulated results under cloudy sky. Thus, I think it would be best that the authors provide some analysis of the clear-sky evaluations (maybe in appendix). This would be helpful for the reader to distinguish the impacts of atmospheric profiles 
and the cloud properties. 4. In part 3: methodology, to derive the necessary cloud property inputs for RTM, the authors also make quite a few assumptions. Especially in deriving the effective radius (Line 145), the used definition is somewhat different from those normally used in parameterization. As the effective radius is a very important quantity that decides the cloud optical properties in the parameterization, the authors need to analyze how the differences in the definition of effective radius will influence the results. 5. There are quite a few places in the text that are not clearly stated and are difficult to understand. For example: Line 301: It is not clear how the probability and cumulative probability are calculated here. And how do you "obviously" figure out from Figure 7 that "total cloud is overestimated in ERA5 and MERRA-2" ? Line 348: How do you define "ratio of the simulation-to-observation frequency of pixels with particular BTs"? Line 353: What does TCC mean? Line 376-377: How do you define mean error (MBTD) and standard error (SBTD) ? 6. Figure captions in this paper are not clear enough to show what the figures are about. For example, Figure 7 "Probability and cumulative probability density for the observed and simulated results ..." - what kind of "results" do you have here? The authors failed to state the name of the variable. Figure 8 "... The results are from Figure 4 marked by blue dashed lines" - couldn't see the "blue dash line" in Figure 4, and actually, there are too many elements in Figure 4.

Minor problems: Line 33: "The ERA5 reanalysis is found the most capability ..." should be "The ERA5 reanalysis is found to have the most capability ..." Line 97: Do you have some references for the CRA-interim? Line 142: "Ignore the uncertainties ..." should be "Ignoring the uncertainties ..."; In addition, is it reasonable to assume mixed phase cloud can be ignored? Line 187: "The correlation between the two is small." - This sentence is vague, as it is not clear about what are "the two". Line 191: "We notice that ..." should be "It is noted that ..." Line 215-217: The authors mentioned the cloud scattering properties in the CRTM are recalculated. Then some necessary validation and description are needed to prove the validity of the new implementation. Line 230: "From" should be "from" Line 272: "with a mean BTs of ..." should be "with a mean BT of ..." Line 324-325: "an abnormal excessive cloud mixing ratio" should be "an 
abnormally excessive cloud mixing ratio" Line 373: "as marked in region A in Figure A"

- where is Figure A? Line 390: "the in-site observation"? Line 413: "demonstrate that AMTD

..." should be "demonstrating that ..."

Interactive comment on Atmos. Meas. Tech. Discuss., doi:10.5194/amt-2019-223, 2019.

Interactive

comment 